\title{
Formation of New Bone with Preferentially Oriented Biological Apatite Crystals Using a Novel Cylindrical Implant Containing Anisotropic Open Pores Fabricated by the Electron Beam Melting (EBM) Method
}

\author{
Takayoshi NAKANO, ${ }^{1)}$ Wataru FUJITANI, ${ }^{1)}$ Takuya ISHIMOTO, ${ }^{11}$ Jee-Wook LEE, ${ }^{11}$ Naoko IKEO, ${ }^{21}$ \\ Hidetsugu FUKUDA ${ }^{3)}$ and Kouichi KURAMOTO ${ }^{3)}$
}

1) Division of Materials and Manufacturing Science, Graduate School of Engineering, Osaka University, 2-1 Yamada-Oka, Suita, Osaka 565-0871 Japan. E-mail: nakano@mat.eng.osaka-u.ac.jp 2) Graduate Student, Division of Materials and Manufacturing Science, Graduate School of Engineering, Osaka University, 2-1 Yamada-Oka, Suita, Osaka 565-0871 Japan. 3) R \& D Group, Nakashima Medical Co., Ltd., 688-1 Joto-Kitakata, Higashi-ku, Okayama 709-0625 Japan.

(Received on September 29, 2010; accepted on November 1, 2010)

\begin{abstract}
New cylindrical bone implants containing elongated pores interconnected as open pores were fabricated by an electron beam melting (EBM) method using Ti-6mass\%Al-4mass\%V ELI powder (mean particle diameter, $65 \mu \mathrm{m}$ ). New bone formation in the elongated pores of the implant and preferential arrangement of biological apatite $c$-axis were confirmed along the long bone axis by microbeam X-ray diffraction. Bone mass and preferential degree of biological apatite $c$-axis, which were considered a bone quality parameter, decreased with the distance from the edge of the implant along the longitudinal bone axis because of a stressshielding effect. Although clear interconnections between new bones appeared through the implant with elongated pores parallel to the bone axis, the defective portion was not covered with new bone in the absence of the new implant.
\end{abstract}

KEY WORDS: electron beam melting; EBM; rapid prototyping; microbeam X-ray diffraction; metal implant; Ti-6Al-4V; bone; apatite; texture; bone quality.

\section{Introduction}

The electron beam melting (EBM) method is a rapid prototyping technique that is adopted for artificially fabricating arbitrarily shaped materials ranging from non-porous materials to porous materials. Materials are produced in crosssectional layers using a three-dimensional (3D) computer model. ${ }^{1,2)}$ Even when metal powder is utilized as a starting material, the use of an electron beam as a heat source for melting the powder facilitates rapid fabrication of laminated structures layer by layer. The use of an electron beam also facilitates control of the pore size, shape, distribution, and porosity during the fabrication of porous materials. Furthermore, since it is possible to fabricate materials directly from metal powder without using a binder, the EBM method is extremely effective for fabricating biological implant metals. ${ }^{3)}$ Therefore, it is possible to fabricate porous metal implants with various 3D morphologies more precisely using this method than using the general melting process, which depends on spontaneous pore formation. Apparent Young's modulus of porous materials can be controlled by varying the configuration of their porous body. By using the EBM method, bone-substitute implants can be developed while preventing the stress-shielding effects aris- ing from the differences between the elastic modulus of the implant metal (e.g., $110 \mathrm{GPa}$ for a Ti-6mass $\% \mathrm{Al}-4 \mathrm{mass} \% \mathrm{~V}$ alloy) and that of living bone (e.g., 15-30 GPa in the longitudinal direction of the cortical bone).

Bone has a hierarchical structure, and hence the mechanical property is controlled at various scales. Bone contains water, minor proteins, and cells, but its main components are type I collagen and fine crystals of biological apatite (BAp); the combination of these two components provides both strength and flexibility to bone. BAp has a hexagonal ionic crystal with strong anisotropic arrangement of ions, and its $c$-axis direction is consistent, ${ }^{4)}$ to a certain degree, with the direction in which the collagen fiber arrranges. ${ }^{5)}$ These facts indicate that the formation of the texture from the BAp/collagen composite in bone tissue is extremely important for defining basic bone function, including the mechanical function. ${ }^{6}$ An assessment carried out by microbeam X-ray diffraction revealed that the BAp $c$-axis orientation of bone depends on the bone shape, site, and in vivo stress distribution. ${ }^{7)}$

In the case of extensive bone defects due to diseases or bone damage due to accidents, bone function can be regained by replacement using grafting implants or regenerative techniques. ${ }^{8,9)}$ Although bone mass or bone density can 
be recovered, there is a significant delay in the recovery of the crystallographic orientation of BAp. Thus, the degree and distribution of BAp crystallographic orientation can be regarded as one of the indicators of bone quality. This delay occurs even when the most advanced regenerative technique is applied, and the delayed recovery of defective bone also has a significant influence on the mechanical function of the bone. ${ }^{10-12)}$

The concept of bone quality was introduced in 2000 by the National Institutes of Health (NIH), and bone quality is related to factors other than bone density that control bone strength. ${ }^{13)}$ When this concept was introduced, several factors such as the bone microstructure represented by the trabecular structure of cancellous bone, formation or restoration of microcracks, state of collagen, bone turnover, and cell viability were considered as potential indicators of bone quality. However, the primary factor influencing bone quality has not yet been fully elucidated. ${ }^{14-16)}$ The orientation of BAp/collagen in a certain direction, which determines the anisotropy of bones at the nanoscale, is also considered as a potential indicator.

In long bones, BAp/collagen has a uniaxial orientation along the longitudinal bone axis. ${ }^{6,7,17)}$ The results of a recent study indicate that grafting anisotropic porous implants with uniaxial elongated pores, including lotus-type porous metal implants, into defective portions of bone tissue with an original uniaxial anisotropic structure in the same direction improved conductivity and had a positive effect on BAp orientation in new bones. ${ }^{18,19)}$ In the case of lotus-type porous metal implants with elongated pores in only one direction, mutual movement of in vivo fluid or tissues, including bone marrow fluid and nutrient vessels, to and from neighboring pores is difficult. This limits normal osteogenesis.

In this study, we aimed to fabricate a new directional implant with an interconnected pore structure. The implant consists of a Ti-6mass $\% \mathrm{Al}-4 \mathrm{mass} \% \mathrm{~V}$ alloy formed by the EBM method. The bone recovery behavior was observed when the implant was grafted into the remodelable long bone in rabbits, focusing on BAp preferential orientation.

\section{Experimental Procedure}

\subsection{Fabrication Process of Implant Specimens}

Ti-6mass\%Al-4mass\%V ELI alloy powder (Arcam, Sweden), which was produced by the gas-atomization process, was used as a starting material for the bone-regenerating implant. The particle diameter of the powder ranged from 45 to $100 \mu \mathrm{m}$ with a mean diameter of $65 \mu \mathrm{m}$, and the average oxygen content was below 1300 mass ppm: these parameters satisfied the American Society for Testing and Materials (ASTM) standard: Ti-6mass $\% A 1-4 m a s s \% V$ ELI material, dissolved oxygen $(\underline{\mathrm{O}}) \leq 1300$ mass ppm. Moreover, the $\underline{\mathrm{O}}$ after EBM was also below 1300 mass ppm, conforming to the standard for an ELI material. An EBM device (Arcam, EBM S12; vacuum: $10^{-2} \mathrm{~Pa}$ ) was used to fabricate a novel Ti-base alloy implant by repeatedly laminating the powder and melting it using electron beam scanning at $100-\mu \mathrm{m}$ intervals based on the CAD model shown in Figs. 1(b)-1(d). The CAD model was cylindrical, $9 \mathrm{~mm}$ in diameter, and $9 \mathrm{~mm}$ in height, a size that can be grafted into (a)

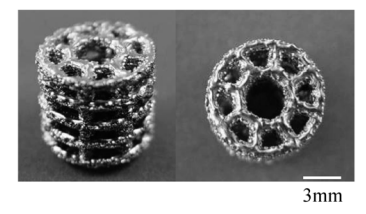

(c)

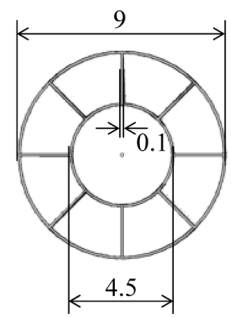

(b)

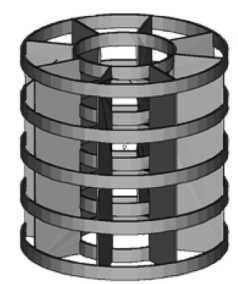

(d)

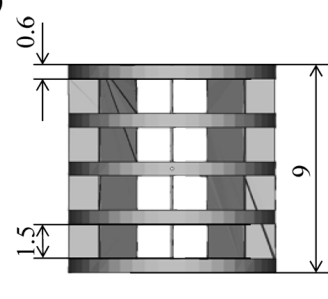

Fig. 1. A novel cylindrical implant with elongated open pores fabricated by the electron beam melting method; (a) morphology of the implant fabricated, (b-d) CAD models designed. The unit is $\mathrm{mm}$.

a rabbit ulna. Since the BAp $c$-axis shows uniaxial orientation along the longitudinal direction in the rabbit ulna, ${ }^{7)}$ the model was designed to have interconnected pores so that it has a through hole in the height direction of the cylindrical implant. In addition, the pores are connected to each other in the vertical direction. The shape parameters of the implant, such as the thickness of the solidified part (grate) and the pore size, were measured using calipers at eight points, and the mean and standard deviation were calculated.

\subsection{Grafting of Implant Specimen into Defective Bone and Measurement of Bone Mass}

A 10-mm-long defect was introduced at the center of the ulna in a male rabbit (Kb1: NZW) so that the fabricated implant could be grafted into the left-forearm ulna. The implant was sterilized for $20 \mathrm{~min}$ at $121^{\circ} \mathrm{C}$ in the autoclave before implantation. The same bone defect was also introduced in the right-forearm ulna, which was used as a control for the state of bone regeneration. After 12 and 24 weeks of regeneration following the grafting of the implant, the forearm radius and ulna were simultaneously extracted, and the tissues were fixed in a $10 \%$ neutral buffered formalin solution. An evaluation of the bone mass was performed using an X-ray radiograph (XIE series; CHUBU MEDICAL Co., Ltd.) and micro-focus X-ray computed tomography ( $\mu$ CT; SMX-100CT-SV; Shimadzu Co., Ltd.; voxel size was set to $39 \mu \mathrm{m}$ per side). 3D bone morphology model was reconstructed using the $3 \mathrm{D}$ visualization software (VGStudio; Shimadzu Co., Ltd.) based on $\mu \mathrm{CT}$ imaging. After implantation, a bone sample was cut from the proximal part (Prox., elbow side) to the distal part (Dist., wrist side) perpendicular to the bone axis (see the inserted figure in Fig. 8) with a band saw of 200- $\mu$ m thickness (Diamond Precision Cutting Systems, BS-300CP, EXAKT), and then the sample was mechanically wet-polished with a 2000 -grit emery paper embedded with $\mathrm{SiC}$ particles. All animal studies were approved by the Animal Care Committee at the Laboratory Animal Center of Osaka University Graduate School of Engineering. 


\subsection{Analysis of BAp Crystal Orientation}

The orientation of the BAp crystals in regenerated bones was analyzed with a microbeam X-ray diffractometer (M18XHF ${ }^{22}$-SRA, Cu K $\alpha, 3.6 \mathrm{~kW}$, Mac Science, or current Bruker AXS). This method is especially powerful to analyze the $c$-axis orientation of BAp and provides information on bone quality for various bones, such as normal, regenerative, and diseased bones. In this study, bone surfaces in the region of $250 \mu \mathrm{m} \phi$ was analyzed with parallel incident Xray beams focused down to $50 \mu \mathrm{m} \phi$ by a metal collimator. The intensities of diffracted X-rays were averaged by swinging and rotating samples along three axes of the crystal under fixed conditions, allowing the detection of reflections in the region where the normal vector to the (002) and (310) diffraction surface in the BAp crystals was tilted from that to the sample surface by 10 degrees or less. All diffracted X-rays, including asymmetric reflections, were detected at the various scattering angle of $2 \theta$, with a curved position sensitive proportional X-ray counter (PSPC). The measurement time was set at $4000 \mathrm{~s}$. The experimental conditions have been described in detail in a previous paper. ${ }^{7)}$

\section{Results and Discussion}

\subsection{Fabrication of Cylindrical Unidirectional Porous Implant}

Optimization of the fabrication conditions permitted complete melting of the grating part and fabrication by electron beam of a new cylindrical unidirectional porous implant with a sufficiently narrow solidified part (Fig. 1(a)). The parameters for optimal fabrication were: accelerating voltage, $60 \mathrm{kV}$; beam current, $2 \mathrm{~mA}$; beam scan rate, $100 \mathrm{~mm} / \mathrm{s}$; and lamination pitch, $0.1 \mathrm{~mm}$ with the focus current and the beam offset derived from the CAD data set at $0 \mathrm{~mA}$ and $0 \mathrm{~mm}$, respectively, to maximally minimize the beam diameter. The fabricated Ti-alloy implant (see Fig. (b)) had a grating wall thickness of $0.73 \pm 0.11 \mathrm{~mm}$ in a radial direction, $3.95 \pm 0.12 \mathrm{~mm}$ pore size at the central hole, $1.30 \pm 0.09 \mathrm{~mm}$ at the surrounding fan-shaped hole, and $9.50 \pm 0.08 \mathrm{~mm}$ diameter of the cylindrical implant. The electron beam width, calculated from the thickness of the grating wall, was over $0.1 \mathrm{~mm}$. Thus, the beam-affected zone was large, suggesting that a melted part measuring over $0.5 \mathrm{~mm}$ was formed during fabrication. If the beam current is lower than or the scan rate is higher than the optimal fabrication parameters used in this study, the implant cannot demonstrate sufficient strength because cracks often appear along the interface between the stacked layers.

Furthermore, because the average size of pores in the cylindrical longitudinal direction was $0.85 \pm 0.05 \mathrm{~mm}$ and the height of the cylindrical implant was $9.19 \pm 0.13 \mathrm{~mm}$, its error was as low as $2 \%$ even though 90 laminations were formed at a pitch of $0.1 \mathrm{~mm}$. This finding implies that this condition was optimal for connecting the solidified parts of each lamination through melting. Moreover, minimal energy input was required for melting and subsequent solidification in the height direction. In this study, the grafting implant was tested in an animal model, i.e., the ulna of a rabbit, using a new unidirectional porous implant with a cylindrical external shape fabricated in the above manner.
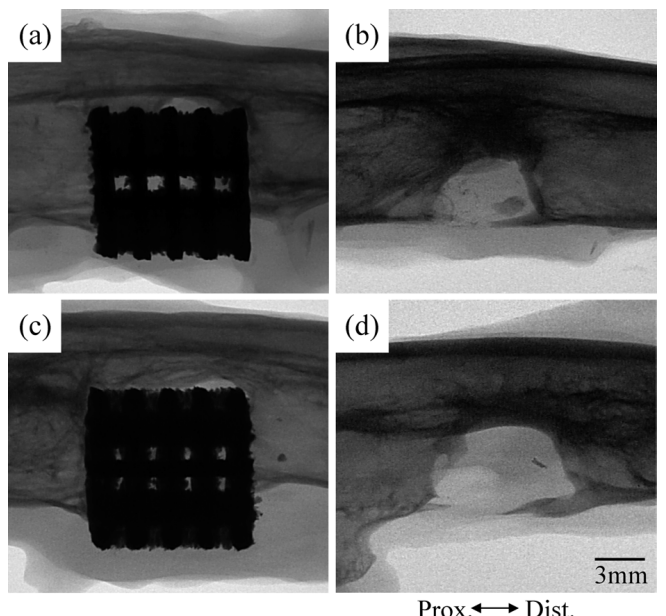

Fig. 2. Radiographs of (a) defected ulnar portion with the implant at 12 weeks after operation, (b) defected portion without the implant at 12 weeks, (c) defected portion with the implant at 24 weeks, and (d) defected portion without the implant at 24 weeks

\subsection{Evaluation of Bone Regeneration Process by Grafting Cylindrical Porous Implant}

Figure 2 shows radiographs of an extracted bone 12 weeks (Figs. 2(a) and 2(b)) and 24 weeks (Figs. 2(c) and 2(d)) after the introduction of the bone defect. Since the ulnar and radial parts are arranged in parallel and the periosteum is packed in between both parts, ${ }^{20}$ the remaining bone shown in the top panel of the photographs is the radius. The cylindrical Ti-base alloy implant that was optimized in the previous section was retained in the defective bone part without any breakage throughout the experimental period. The absence of a bone defect in the bone/implant interface indicated that bone regeneration was progressing favorably. Although osteoid tissues were observed in the proximity of the radius at 12 weeks in the control group in which no implant was grafted, the defective part was not covered by new bones. At 24 weeks it again showed a clarified bone defect, indicating no connection of the defective part with new bone. Moreover, $\mu \mathrm{CT}$ imaging (Fig. 3) reveals that when the cylindrical Ti-base alloy implant was not grafted, there was only an increase in bone width in the cortical part of the radius even 12 weeks after the introduction of the bone defect, with no regeneration of ulna observed although regeneration was expected based on the radiograph in Fig. 2(b). In addition, pore formation and sponging of the cortical bone was apparent in the proximity of the regenerated bone, suggesting the formation and existence of immature bone. In general, when the release of the cytokines involved in bone formation is insufficient because of a massive defect, the bone regenerates to a certain degree at an early stage. ${ }^{21)}$ If there is insufficient stress transfer to the osteocyte that is believed to function as a mechanosensor sensing any stress, the result is a difficult bone union. ${ }^{22}$ ) Furthermore, a clear change in the shape of the bones, including the radius, was observed even around the bone defect installation, possibly as a result of the change in the in vivo stress field even in the control group, which did not undergo grafting of an implant.

The formation of new bones in the implant is illustrated 

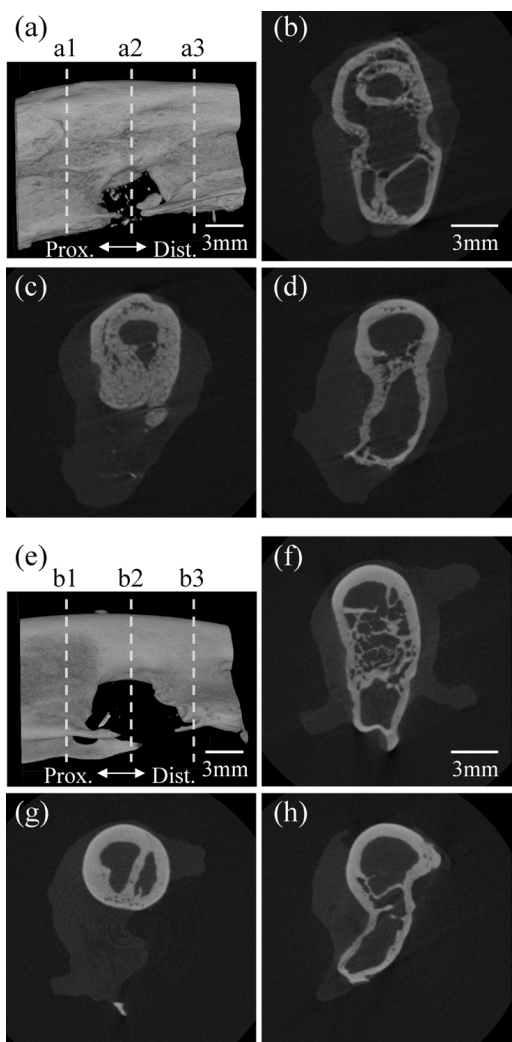

Fig. 3. MicroCT images of defected portions without the implant at 12 weeks (a-d) and at 24 weeks (e-h). (a) is a 3dimensional (3D) reconstructed image showing cross sections, a1, a2 and a3, corresponding to (b), (c) and (d), respectively. (e) is a $3 \mathrm{D}$ reconstructed image showing cross sections, b1, b2 and b3, corresponding to (f), (g) and (h), respectively.
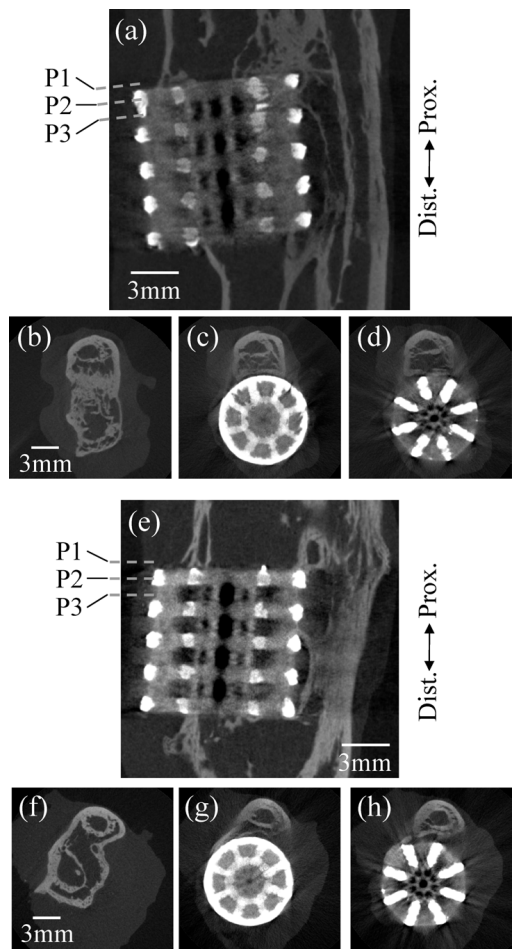

Fig. 4. Radiographs of defected portions at 12 weeks (a-d) and at 24 weeks $(\mathrm{e}-\mathrm{h})$. (a) shows the positions for CT cross sections, P1, P2 and P3, corresponding to (b), (c) and (d), respectively. (e) shows the positions for CT cross sections, P1, P2 and P3, corresponding to (f), (g) and (h), respectively. in Fig. 4, which shows cross-sections in the 3D reconstruction images formed from the $\mu \mathrm{CT}$ image in the presence of the implant. Although the Ti alloy has a small atomic number and its X-ray absorption coefficient is relatively low, metal artifacts derived from a large difference in the absorption coefficient of new bone/Ti were observed in the section vertical to the bone longitudinal axis, preventing the clear determination of the existence of new bones. When materials with a high X-ray absorption capability, such as metals, are scattered among materials with low X-ray absorption capability or when the X-ray passing distances differ markedly depending on the beam direction, these artifacts are reported to often occur. ${ }^{23)}$ These artifacts were also observed 24 weeks after the introduction of the defect, preventing the accurate determination of the formation of new bones. However, because stress transfer to the surrounding bones, including transfer to the implant edge, is achieved from an early stage of implantation, bone formation proceeds relatively normally.

\subsection{New Bone Formation and Apatite Orientation in the Implant}

CT imaging did not clearly reveal the existence of new bones in the implant. Figure $\mathbf{5}$ presents representative radiographs observed from the direction of the bone axis after they were sliced in the direction perpendicular to the bone axis. The bone sample was sliced from the proximal part (Prox., elbow side) of the ulna to the distal (Dist., wrist side) part with a band saw to expose the cross-sections from P1 to P6 (see the photo in Fig. 8). Radiographs also show the part proximal to the surrounding bone (the part including P1: Figs. 5(a) and 5(d)), the edge of the implant (the part including P2: Figs. 5(b) and 5(e)), and the part near the center of the implant (the part including P5: Figs. 5(c) and 5(f)). In addition to the existence of a radius without the introduction of the defect, clear formation of new
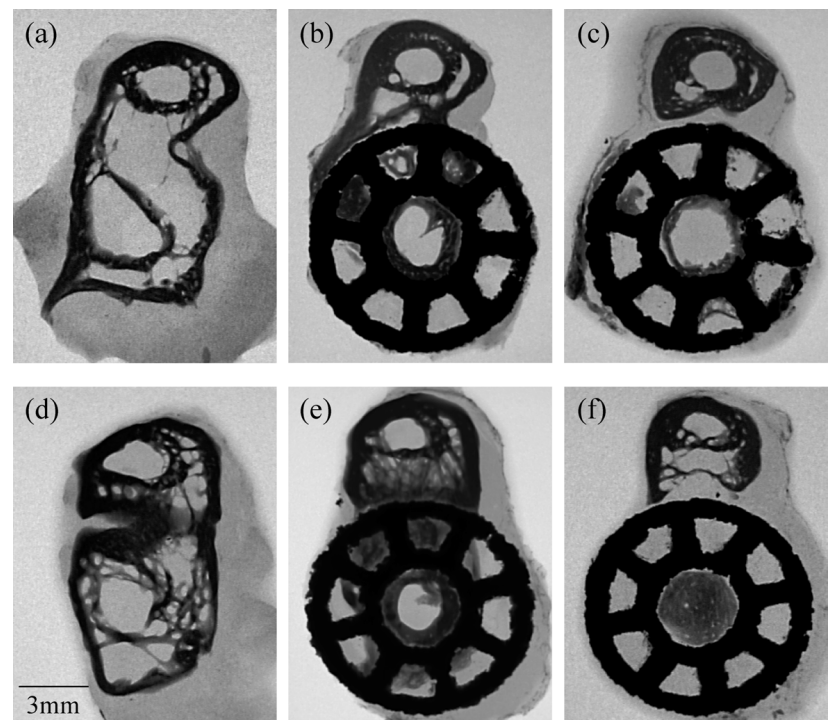

Fig. 5. Radiographs of bone specimens sliced perpendicular to the longitudinal bone axis at 12 weeks $(\mathrm{a}-\mathrm{c})$ and at 24 weeks (d-f); (a) cross section with P1 at 12 weeks, (b) cross section with P2 at 12 weeks, (c) cross section with P5 at 12 weeks, (d) cross section with P1 at 24 weeks, (e) cross section with P2 at 24 weeks, and (f) cross section with P5 at 24 weeks. 


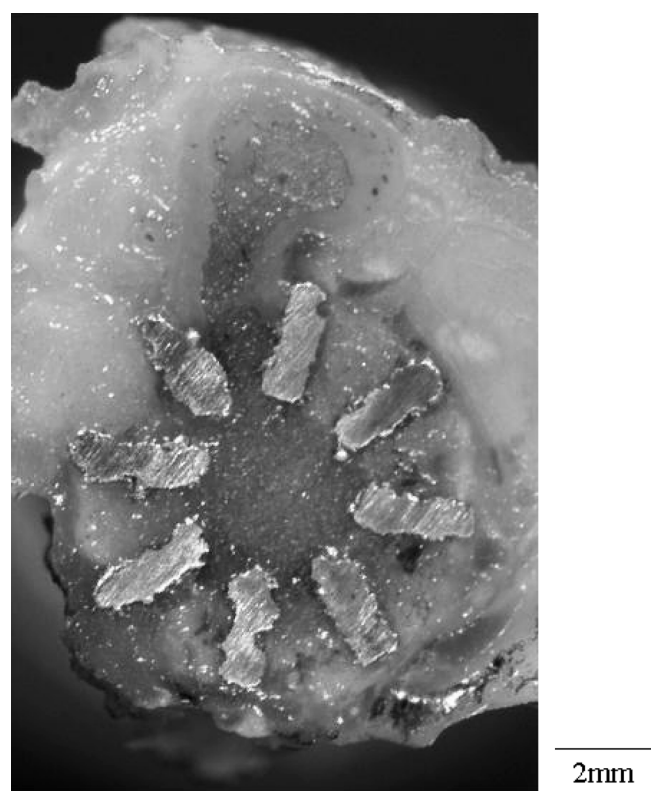

Fig. 6. Optical microscopic image clearly showing medullary cavity near the center of cross section in the implant at 12 weeks.

bones into the cylindrical-shape implant that corresponds to the ulna can be observed. The formation of new bones was observed in the defect from the radial side to the hole at the center of the implant in every age group. Using an optical microscope, the existence of bone marrow was confirmed (Fig. 6). This implies formation of the cortical bone part that has a similar shape to the cylinder. It suggests activation of the bone cells, which are differentiated from the mesenchymal stem cells and blood stem cells, and progression of bone reconstruction because of the existence and retention of bone marrow. ${ }^{24}$

In the hole at the center of the implant, new bone formation along the grating wall was observed. Interconnections between the new bones and the retention of the bone marrow cavity were also found, but there was a decrease in new bone mass from the edge to the center of the implant. This indicates new bone conductivity in the new cylindrical unidirectional porous implant.

Because the formation of new bone was observed in all the sections at the central hole of the implant, the four points $(\bigcirc)$ of the new bone at the central hole and other regions of new bone formation, as shown in Fig. 7, were focused on for analysis of BAp orientation. The result of analyzing the BAp $c$-axis orientation is shown as a measure of bone quality, and hence, it is overlapped with the radiographs. The value of random orientation for BAp was 2, indicating that preferential orientation along the direction of the bone axis was recovered above a value of 2 . Figure 7 shows the apatite orientation in the P2 section in the proximity of the implant edge (see the photo in Fig. 8). The existence of $c$-axis orientation along the bone longitudinal axis was already observed 12 weeks after the introduction of the bone defect, and further preferential alignment of BAp through remodeling proceeded at 24 weeks, showing a degree of orientation up to 14 . The BAp orientation in the mature rabbit ulna is about $20,{ }^{7,11)}$ revealing a clear recovery of BAp orientation along the bone longitudinal axis, al-
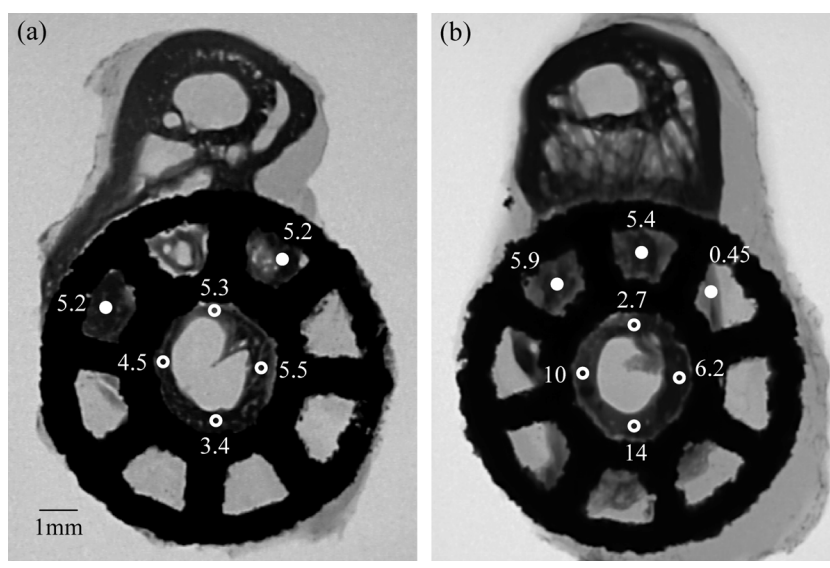

Fig. 7. Preferential degree of biological apatite $c$-axis orientation depending on the position in the cross section of $\mathrm{P} 2$ at 12 weeks (a) and at 24 weeks (b). Four open circles (O) show the position analyzed by the microbeam X-ray diffraction method in Fig. 7.

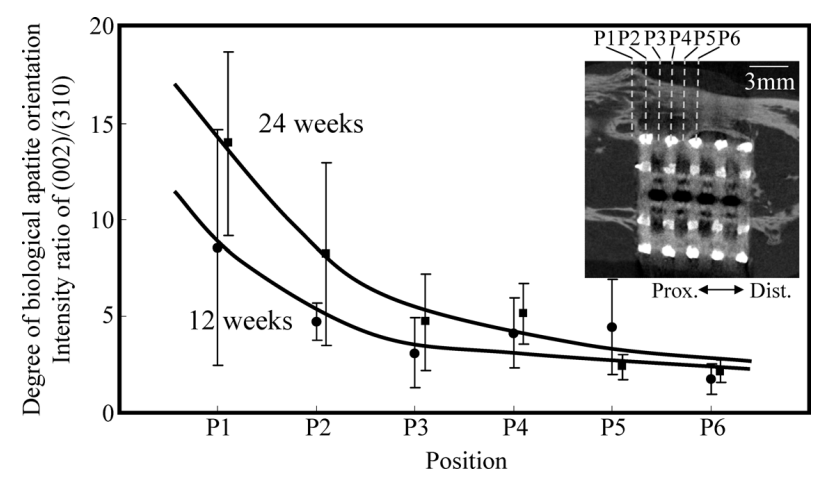

Fig. 8. Degree of biological apatite $c$-axis orientation as a function of position between P1 and P6 at 12 weeks and at 24 weeks after operation. Analyzed positions, P1, P2, P3, $\mathrm{P} 4, \mathrm{P} 5$ and $\mathrm{P} 6$, are indicated in the inserted radiograph.

though the degree of orientation was slightly low even at 24 weeks.

Figure 8 shows the site-dependence of the $c$-axis orientation of BAp along the bone longitudinal axis that was analyzed in the four new bone parts $(\bigcirc)$ (the mean and standard deviation of the four points) shown in Fig. 7. Although a rapid decrease in the degree of orientation was observed from the edge (P2) to the center (P6) of the implant, the microbeam X-ray diffraction profile confirmed the formation of BAp crystals, i.e., normal bone regeneration. It is concluded that the new implant induced bone regeneration because the control group did not show any bone regeneration at the center of the bone.

Although the bone proximal part showed a trend toward apatite orientation recovery in the implant with an increase in the grafting period, there was virtually no increase in orientation around the center of the implant, with observed orientation degrees being as low as 4 to 5 . It also shows good conformity to the trend of decreasing amounts of new bone formed at the center of the implant (Fig. 5). It is clear that the decrease in bone mass and apatite $c$-axis orientation was caused by a stress-shielding effect ${ }^{25}$ ) and because the formation of new bone was initiated at the bone defect/implant interface. The stress-shielding effect is a phenomenon whereby bone mass or bone quality decreases when certain 
materials such as metals with a high Young's modulus are placed in parallel with bone tissue with a low Young's modulus. It is closely related to the function of osteocytes, stress-sensitive cells existing in bone tissue. When the stress-shielding effect is noted, although Young's modulus apparently decreases in the new implant, the stress transferred to the new bone shows a greater decrease in cases with a metal implant than in cases without the metal implant. Hence, it is concluded that bone mass and BAp orientation were decreased because of a decrease in the stress transfer to the osteocyte compared to that seen in the normal part. However, the fact that the center of the implant also showed BAp-preferential orientation along the bone longitudinal axis, albeit to a lesser degree, combined with the progression of the bone connection or bone marrow formation promises the long-term retention of bones within the implant. When an implant with a conventional lotus pore was grafted in an animal, the orientation along the new bone axis not only decreased toward the center of the implant but also showed a decreasing trend even at 13 weeks because of the increased duration. ${ }^{18)}$ In this study, because the implant is composed of pores interconnected not only in the direction of the bone axis but also in the vertical direction, there is no decrease in orientation with increasing grafting period. As a result, it was deemed possible to supply oxygen and nutrients to the inside of the porous implant with adequate vascular access ${ }^{26)}$ and to have bone-marrow fluid flow and cell migration. ${ }^{27)}$

Porous materials are known to potentially have bone conductivity. In other words, it is possible to facilitate bone formation in the bone-defect part by grafting porous materials into that part without administering growth factors or stem cells. ${ }^{28)}$ This study also demonstrated that a new bone was formed at the center of the defect, which does not commonly allow the formation of bones. Adhesive cells such as osteoblasts continue to function by adhering to materials that provide a foothold via proteins such as integrin. Cell adhesion directly affects cell growth, differentiation, and migration and the resultant tissue morphology or regeneration process. ${ }^{29)}$ When cells cannot secure a foothold for adhesion, they cannot exert their function. Accordingly, providing the bone-defect part with porous material in a form that serves as a foothold presumably allows cell migration and new bone formation in different types of defective parts.

Furthermore, bone formation in porous materials is known to be closely related to the pore size. Previous studies have indicated that a pore size of $100-400 \mu \mathrm{m}$ is optimal or that $100 \mu \mathrm{m}$ is not the lower limit. ${ }^{30,31)}$ The implant used in this study had a pore size of $3.95 \pm 0.12 \mathrm{~mm}$ at the central hole, where the formation of a bone marrow cavity was observed, showing a value much higher than the optimal value. Meanwhile, the formation of new bones and bone connections, which permit the retention of the bone marrow, were observed. Therefore, this study suggests that it is important to graft the implant so that the elongation direction of the anisotropic pores in the unidirectional implant is consistent with the oriented direction of the long bone with uniaxial orientation. This study also suggests that the new implant plays an important role as a foothold for the formation of bone interconnections during cell migra- tion. $^{32)}$

\section{Conclusion}

We fabricated a new unidirectional porous implant made of a Ti-6mass $\% \mathrm{Al}-4 \mathrm{mass} \% \mathrm{~V}$ alloy using the EBM method; this implant can be grafted into bones. We performed grafting in rabbits, and we conducted bone regeneration in the new implant, with a focus on the bone mass and bone quality of new bones. Our conclusions are as follows:

(1) It was possible to fabricate a unidirectional porous implant for bone replacement by using Ti-6mass\%Al$4 \mathrm{mass} \% \mathrm{~V}$ powder as a starting material and using the EBM method, or an equivalent method for rapid prototyping. A new implant with a grating width of $0.7 \mathrm{~mm}$ was successfully fabricated; thus, unidirectional open pores were introduced in the direction of the cylindrical axis and pores were also connected in the vertical direction.

(2) The unidirectional porous implant connected the defective part with new bone by forming a bone-marrow cavity at the center and cortical bones in the surrounding area and, in addition, forming preferential alignment of BAp in the direction of the bone longitudinal axis. In contrast, when the implant was not grafted, it was difficult to regenerate the defective ulna.

(3) There was a decrease in the new bone mass and the $c$-axis orientation of apatite along the bone longitudinal axis from the edge to the center of the implant. This was attributed to the stress-shielding effect and to the fact that the new bone formation starts from the cross-section of the bone. Nevertheless, there was no bone resorption even after 24 weeks, and the BAp $c$-axis orientation along the bone longitudinal axis was observed even at the center of the implant, albeit to a lesser extent.

This finding may be attributed to the fact that this implant has open pores not only in the direction of the cylindrical axis but also in the vertical direction, and this is favorable for bone-marrow fluid flow or cell migration.

As shown above, the new cylindrical-shape implant with elongated open pores fabricated in this study functioned as a foothold for new bone transfer along the direction of the bone longitudinal axis; as a result, in the implant, the new bones were connected along the bone longitudinal axis, and the preferential alignment of BAp was dependent on the bone position. The new implant facilitates long-term retention of the bone connection without eliciting bone resorption.

\section{Acknowledgments}

This study was performed with the support of the iron and steel research promotion grant from the Iron and Steel Institute of Japan, Grants-in-aid from the global COE program "Center of Excellence for Advanced Structural and Functional Materials Design" (Osaka University), the Research Grant Program of the Ministry of Education, Culture, Sports, Science and Technology, the "Super Special Consortia" for the supporting development of cutting edge medical care (Cabinet office, Government of Japan, and Ministry of Health, Labor and Welfare, Ministry of Education, Culture, Sports, Science and Technology, Ministry of Economy, Trade and Industry) and the Light Metal Educa- 
tional Foundation, Inc.

\section{REFERENCES}

1) G. Ryan and A. Pandit: Biomaterials, 27 (2006), 2651.

2) M. Melican, M. Zimmerman, M. Dhillon, A. Ponnambalam, A. Curodeau and J. Parsons: J. Biomed. Mater. Res. A, 55 (2001), 194.

3) M. Niinomi Ed.: Metals for Biomedical Devices, Woodhead Publishing, Oxford, (2010), 355.

4) J. C. Elliot: Structure and Chemistry of the Apatites and Other Calcium Phosphates, Elsevier, Amsterdam, (1994), 191.

5) W. J. Landis, M. J. Song, A. Leith, L. McEwen and B. F. McEwen: J. Struct. Biol., 110 (1993), 39.

6) T. Nakano, Y. Tabata and Y. Umakoshi: Texture and Bone Reinforcement. Encyclopedia of Materials: Science and Technology-Updates, Ms2061, eds. by K. H. J. Buschow, R. W. Cahn, M. C. Flemings, E. J. Kramer, S. Mahajan, P. Veyssiere, Elsevier, Oxford, (2005), 1.

7) T. Nakano, K. Kaibara, Y. Tabata, N. Nagata, S. Enomoto, E. Marukawa and Y. Umakoshi: Bone, 31 (2002), 479.

8) Y. Tabata, K. Yamada, S. Miyamoto, I. Nagata, H. Kikuchi, I. Aoyama, M. Tamura and Y. Ikada: Biomaterials, 19 (1998), 807.

9) O. L. A. Harrysson, O. Cansizoglu, D. J. Marcellin-Little, D. R. Cormier and H. A. WesII: Mater. Sci. Eng. C, 28 (2008), 366.

10) T. Nakano, K. Kaibara, Y. Tabata, N. Nagata, S. Enomoto, E. Marukawa and Y. Umakoshi: Proc. of TETU-6, Elsevier, Amsterdam, (2002), 95.

11) T. Ishimoto, T. Nakano, Y. Umakoshi, M. Yamamoto and Y. Tabata: Mater. Sci. Forum, 512 (2006), 261.

12) T. Nakano, T. Ishimoto, J.-W. Lee and Y. Umakoshi: J. Ceram. Soc. Jpn., 116 (2008), 313.

13) NIH Consensus Development Panel on Osteoporosis Prevention, Diagnosis, and Therapy, Osteoporosis Prevention, Diagnosis, and Therapy, JAMA, 285 (2001), 785.

14) M. Ito, K. Ikeda, M. Nishiguchi, H. Shindo, M. Uetani, T. Hosoi and H. Orimo: J. Bone Miner. Res., 20 (2005), 1828.

15) M. Saito, K. Fujii, S. Soshi and T. Tanaka: Osteoporos. Int., 17
(2006), 986.

16) N. Sakagami, N. Amizuka, M. Li, K. Takeuchi, M. Hoshino, M. Nakamura, K. Nozawa-Inoue, N. Udagawa and T. Maeda: Micron, 36 (2005), 688

17) N. Sasaki, N. Matsushima, T. Ikawa, H. Yamanura and A. Fukuda: $J$. Biomech., 22 (1989), 157.

18) T. Nakano, T. Kan, T. Ishimoto, Y. Ohashi, W. Fujitani, Y. Umakoshi, T. Hattori, Y. Higuchi, M. Tane and H. Nakajima: Mater. Trans., 47 (2006), 2233

19) K. Alvarez, S.-K. Hyun, T. Nakano, Y. Umakoshi and H. Nakajima: Mater. Sci. Eng. C, 29 (2009), 1182.

20) P. Kasten, J. Vogel, F. Geiger, P. Niemeyer, R. Luginbühl and K. Szalay: Biomaterials, 29 (2008), 3983.

21) J. P. Schmitz, Z. Schwartz, J. O. Hollinger and B. D. Boyan: Acta Anat., 138 (1990), 185.

22) B. S. Noble and J. Reeve: Molecul. Cell. Endocrinol., 159 (2000), 7.

23) R. Popilock, K. Sandrasagaren, L. Harris and K. A. Kaser: J. Nucl. Med. Tech., 36 (2008), 79.

24) I. F.-T. Hernández-Gil, M. A. A. Gracia, M. C. Pingarrón and L. B. Jerez: Med. Oral. Patol. Oral. Cir. Bucal., 11 (2006), E47.

25) T. M. Turner, D. R. Sumner, R. M. Urban, R. Igloria and J. O. Galante: J. Bone Jt. Surg., 79A (1997), 1381.

26) D. R. Jordan, L. A. Mawn, S. Brownstein, T. M. McEachren, S. M. Gilberg, V. Hill, S. Z. Grahovac and J. P. Adenis: Ophthal. Plast. Reconstr. Surg., 16 (2000), 347.

27) E. Pamula, E. Filová, L. Baáková, V. Lisá and D. Adamczyk: J. Biomed. Mater. Res. A, 89A (2009), 432.

28) T. Steffen, T. Stoll, T. Arvinte and R. K. Schenk: Eur. Spine J., 10 (2001), S132.

29) K. Burridge, K. Fath, T. Kelly, G. Nuckolls and C. Turner: Ann. Rev. Cell Biol., 4 (1988), 487.

30) V. Karageorgiou and D. Kaplan: Biomaterials, 26 (2005) 5474.

31) H. E. Götz, M. Muller, A. Emmel, U. Holzwarth, R. G. Erben and R. Stangl: Biomaterials, 25 (2004) 4057.

32) E. Jabbari: Proc. MRS, 897 (2005), 123. 